\title{
The Frequency of Visually Induced Gamma-Band Oscillations Depends on the Size of Early Human Visual Cortex
}

\author{
D. Samuel Schwarzkopf, ${ }^{1,2}$ David J. Robertson, ${ }^{2}$ Chen Song, ${ }^{1,2}$ Gareth R. Barnes, ${ }^{1}$ and Geraint Rees ${ }^{1,2}$ \\ ${ }^{1}$ Wellcome Trust Centre for Neuroimaging, University College London (UCL) Institute of Neurology, London WC1N 3BG, United Kingdom, and ${ }^{2}$ UCL \\ Institute of Cognitive Neuroscience, London WC1N 3AR, United Kingdom
}

The structural and functional architecture of the human brain is characterized by considerable variability, which has consequences for visual perception. However, the neurophysiological events mediating the relationship between interindividual differences in cortical surface area and visual perception have, until now, remained unknown. Here, we show that the retinotopically defined surface areas of central V1 and V2 are correlated with the peak frequency of visually induced oscillations in the gamma band, as measured with magnetoencephalography. Gamma-band oscillations are thought to play an important role in visual processing. We propose that individual differences in macroscopic gamma frequency may be attributed to interindividual variability in the microscopic architecture of visual cortex.

\section{Introduction}

The surface area of human primary visual cortex (V1), defined by noninvasive retinotopic mapping, varies up to threefold between individuals (Dougherty et al., 2003), with consequences for visual perception (Duncan and Boynton, 2003; Schwarzkopf et al., 2011). The functional architecture of V1 contains a columnar map in which neurons with similar tuning properties (e.g., orientation preference) cluster together (Bonhoeffer and Grinvald, 1991; Hübener et al., 1997; Yacoub et al., 2008). As the size of the cortex increases, either the number or the width of such columns (or both) will increase, although the precise relationship is not yet known. Consistent with this, both the width and number of cortical columns is highly variable across individuals of the same species (Horton and Hocking, 1996). The substantial variability in size of V1 is unlikely to be accompanied by anatomical scaling of the physical size of individual neurons or the length of connections between neurons, suggesting that local intracortical connectivity between neurons may be weaker in individuals with a larger V1. This implies that the perceptual or behavioral effects mediated by these connections are likely to vary with the surface area of V1: in brains with a large V1, these contextual effects should be weaker. Consistent with this hypothesis, V1 surface area correlates inversely with the strength of two visual illusions

\footnotetext{
Received Sept. 19, 2011; revised Dec. 5, 2011; accepted Dec. 22, 2011.

Author contributions: D.S.S. and G.R.B. designed research;D.S.S. and D.J.R. performed research; D.S.S. analyzed data; D.S.S., C.S., G.R.B., and G.R. wrote the paper.

This work was supported by the Wellcome Trust (G.R., D.S.S., G.R.B.), the Medical Research Council (D.J.R.), and the Brain Research Trust (C.S.). We dedicate this study to T. Schofield (1976-2010) and thank him for his help with data collection. We thank K. Singh and D. Pinotsis for helpful discussions of this work.

The authors declare no competing financial interests.

Correspondence should be addressed to D. Samuel Schwarzkopf, WT Centre for Neuroimaging at UCL, 12 Queen Square, London WC1N 3BG, UK. E-mail: s.schwarzkopf@ucl.ac.uk.

DOI:10.1523/JNEUROSCI.4771-11.2012

Copyright $\odot 2012$ the authors $\quad 0270-6474 / 12 / 321507-06 \$ 15.00 / 0$
}

in which the perceived size of objects are influenced by their surrounding context (Schwarzkopf et al., 2011). This interindividual variability in V1 surface area and concomitant changes in local connectivity may also have consequences for the properties of the neuronal population dynamics in visual cortex. A visual stimulus induces a characteristic sustained macroscopic oscillatory response measurable with magnetoencephalography (MEG) in the gamma $(30-80 \mathrm{~Hz})$ band (Adjamian et al., 2004) that may play an important role in sensory and cognitive processing (Herrmann et al., 2010). Most studies on gamma oscillations pertain to oscillatory power; the role of the peak frequency in the gamma response has received far less attention, although some recent studies reported a relationship between gamma peak frequency and behavior (Edden et al., 2009; Muthukumaraswamy et al., 2009, 2010).

Several modeling studies have examined the link between the gamma spectrum and the anatomical structure of V1. These range from continuous-wave models (Robinson, 2006), in which the gamma spectrum is a consequence of this patchy intracortical connectivity; to coupled oscillator models (Hadjipapas et al., 2009), where the shape of the spectrum is determined by local coupling parameters. Indirect empirical evidence for a link between the spatial structure and the temporal dynamics already exists in that orientation tuning has been linked to both homogeneous columnar structure (Nauhaus et al., 2008) and gamma frequency (Edden et al., 2009). We therefore hypothesized that the peak frequency of visually induced gamma activity would vary with V1 surface area. To test this, we measured such signals from visual cortex using MEG and related them to retinotopically determined visual cortical size.

\section{Materials and Methods}

Participants. Sixteen neurologically normal, healthy individuals with normal vision (age, 19-34 years; 6 female; 2 left-handed) participated in 

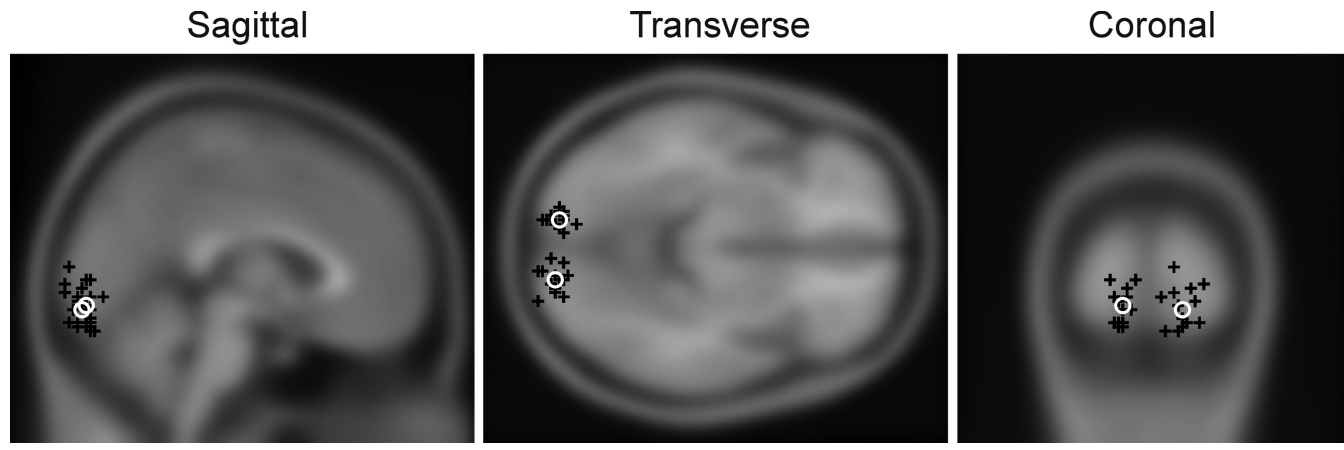

Figure 1. Peak MNI coordinates of the gamma responses induced by the stimulus for all participants overlaid on a canonical structural MRI template brain. Black crosses denote the peak for each individual participant associated with stimulation of the contralateral visual hemifield. White circles denote the mean peak coordinates across participants for stimulation of this hemifield.

two sessions. In the first session, participants were scanned with MEG to record the response in visual cortex to a simple static grating stimulus. In the second session, we performed retinotopic mapping using functional magnetic resonance imaging (fMRI). This retinotopic mapping session also formed part of a previous study (Schwarzkopf et al., 2011). Participants gave written informed consent to take part in the experiments. All procedures were approved by the local ethics committee.

Magnetoencephalography. Participants were seated in a MEG system and viewed the stimulus on a projection screen in front of them. During the entire recording run, they were required to maintain fixation on a small red dot in the center of the screen. The stimulus was a static, high-contrast, square-wave, vertical grating, which measured $4^{\circ} \times 4^{\circ}$ of visual angle and had a spatial frequency of 3 cycles $/{ }^{\circ}$. The grating was presented on a mean luminance uniform gray background in the lower visual field such that the corner closest to the center of gaze was horizontally and vertically displaced from the fixation spot by $0.5^{\circ}$. There were 180 trials per run. On half of the trials, the grating was shown in the lower right quadrant; on the other half, it was shown in the lower left. Moreover, to minimize adaptation effects, the contrast polarity was either positive or negative. These four trial conditions were counterbalanced and randomly interleaved within a run. The stimulus was presented for $1.5-2 \mathrm{~s}$ with the duration pseudorandomized on each trial. To ensure attention and arousal, participants were required to press a button on a response box as soon as the stimulus disappeared. The intertrial interval was fixed at $2 \mathrm{~s}$. On two separate runs, participants made their behavioral response either with their left or right hand. The order was counterbalanced across participants.

We recorded MEG data using a whole-head CTF axial gradiometer system with 275 channels, sampled at $600 \mathrm{~Hz}$. Three electrical coils were placed at fiducial locations and used to monitor subject head movement. Data were analyzed using SPM8 (http://www.fil.ion.ucl.ac.uk/spm). Recordings were divided into epochs that comprised the time from $1.5 \mathrm{~s}$ before stimulus onset until $1.5 \mathrm{~s}$ after stimulus onset (i.e., the earliest time for stimulus offset that preceded the participant's behavioral response). We then used an LCMV beamformer algorithm (Van Veen et al., 1997) implemented in SPM8 to contrast the source power in a time window between 0.5 and $1.5 \mathrm{~s}$ after stimulus onset with the $1 \mathrm{~s}$ of baseline power directly preceding stimulus onset. Source orientation at each voxel was determined using the method of Sekihara and colleagues (2004). We located peak gamma activity in the medial occipital cortex, and at this peak location we used the beamformer weights to extract the time series of the source. Power spectra for frequencies between 30 and $80 \mathrm{~Hz}$ during the stimulation and the baseline time window were calculated using a multitaper spectral estimate (Thomson, 1982) using seven discrete prolate spheroidal sequences as data tapers. We determined the percentage power change in each frequency bin and fitted a Gaussian function to this curve to derive the peak gamma frequency, the amplitude, and the bandwidth of the gamma response. We calculated the mean across all four measurements, i.e., for both visual hemifields and the two separate recording runs. To illustrate the complete time-frequency evolution of the spectral power change, we used a wavelet transform (implemented by the spm_wavspec function in SPM8). For each frequency bin, we determined the percentage power change at each time point relative to the entire $1.5 \mathrm{~s}$ of baseline preceding stimulus onset.

Retinotopic mapping. In the same participants, we conducted retinotopic mapping (Schwarzkopf et al., 2011). Participants were scanned in a Siemens Trio 3T MRI scanner with a 32-channel head coil while they viewed rotating high-contrast checkerboard wedge and expanding/contracting ring stimuli reversing contrast at $\sim 4 \mathrm{~Hz}$ against a uniform gray background. The wedge stimulus subtended eccentricities between $0.3-8^{\circ}$ and a polar angle of $15^{\circ}$. The ring stimuli scaled in width over the course of a stimulus cycle and were $3^{\circ}$ wide at the maximal eccentricity. At random intervals, the checkerboard pattern would undergo a small jump in polar angle and jump back after $200 \mathrm{~ms}$. Participants were asked to indicate whenever this happened with a button press.

An echo-planar imaging sequence (TR, 3.06 s; echo spacing, $560 \times$ $10^{-3} \mathrm{~ms}$; matrix size, $96 \times 96$; interleaved slice acquisition) affording $2.3 \times 2.3 \times 2 \mathrm{~mm}$ resolution $(0.3 \mathrm{~mm}$ interslice gap $)$ was used to acquire functional images. In addition, a T1-weighted modified driven equilibrium Fourier transform sequence was used to generate a structural image of brain anatomy, and $\mathrm{B}_{0}$-field maps were acquired to correct for EPI distortions (Hutton et al., 2002). In polar-mapping sessions, the wedge stimulus made 10 rotations (20 images/rotation). In eccentricitymapping sessions, the ring stimulus made 15 contraction/expansion cycles (10 images/cycle).

Functional magnetic mapping data were preprocessed in SPM8 by applying slice timing correction, realignment, and unwarping (using the $\mathrm{B}_{0}$-field maps). We coregistered the images to the participant's structural MRI scan and smoothed it with a $4 \mathrm{~mm}$ FWHM kernel. We calculated a fast Fourier transform of the time series for each scanning session at the fundamental frequency of the stimulus to determine the stimulus location at which each voxel responded (Sereno et al., 1995). These data were then projected onto a mesh created from the gray-white matter boundary in Freesurfer (Fischl et al., 1999). The borders of early visual areas V1-V3 were delineated based on the reversals in the polar angle map. Surface area in each region was determined for each participant.

\section{Results}

Employing a beamforming approach, we isolated evoked and induced MEG responses to a visual stimulus in a virtual electrode placed in the medial occipital cortex in a location consistent with primary visual cortex. Figure 1 shows the peak for the source localization of the visually induced gamma-band response in all participants (and the mean coordinates across participants) overlaid on a structural MNI template brain. As expected, the sources for a stimulus in a visual hemifield clustered within the contralateral medial occipital cortex. This revealed substantial variability between individuals (Fig. 2) in the peak of stimulus-induced gamma-band oscillations between 44.6 and $57 \mathrm{~Hz}$. Previous studies reported higher gamma frequencies over a wider range (Hoogenboom et al., 2006); however, these experiments used a drifting grating stimulus and moving or dynamic stimuli induce 
A
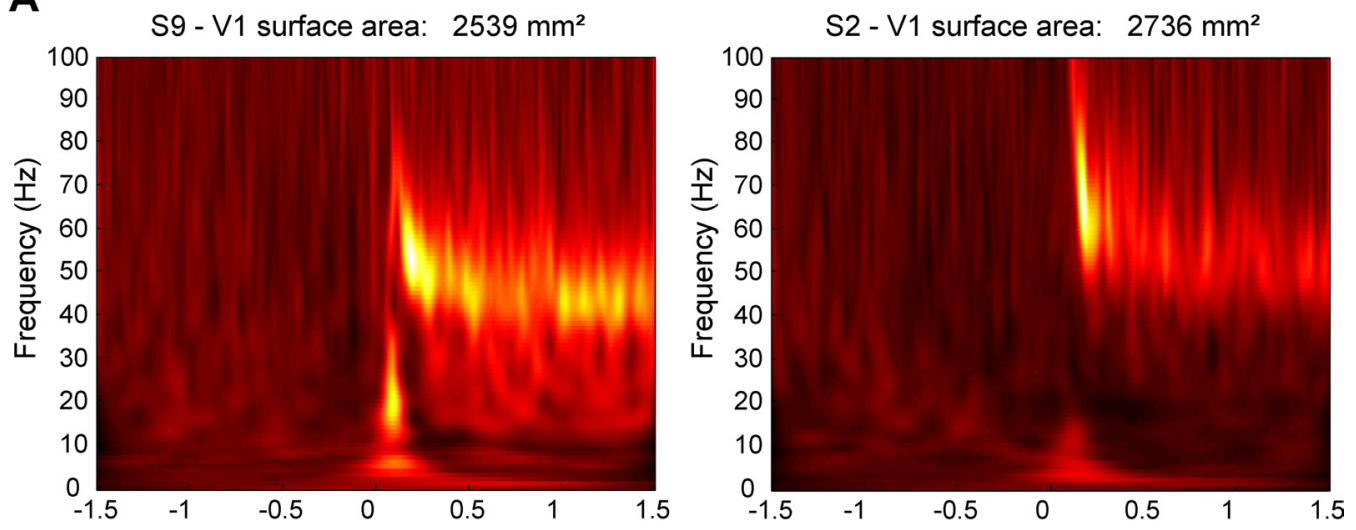

Time relative to stimulus onset (s)

B

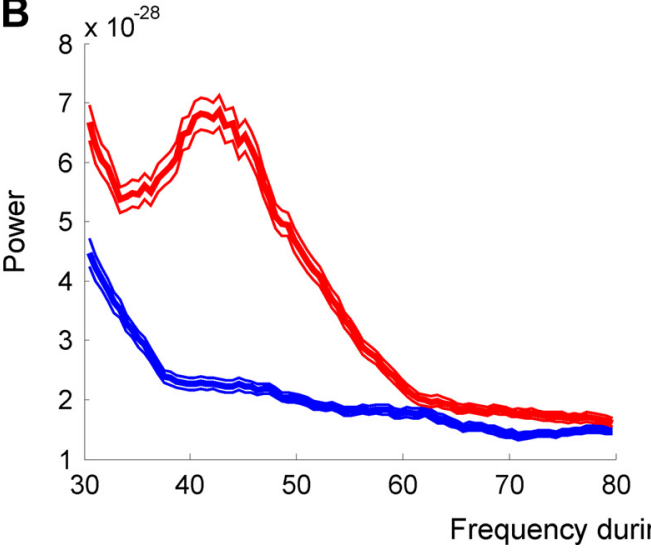

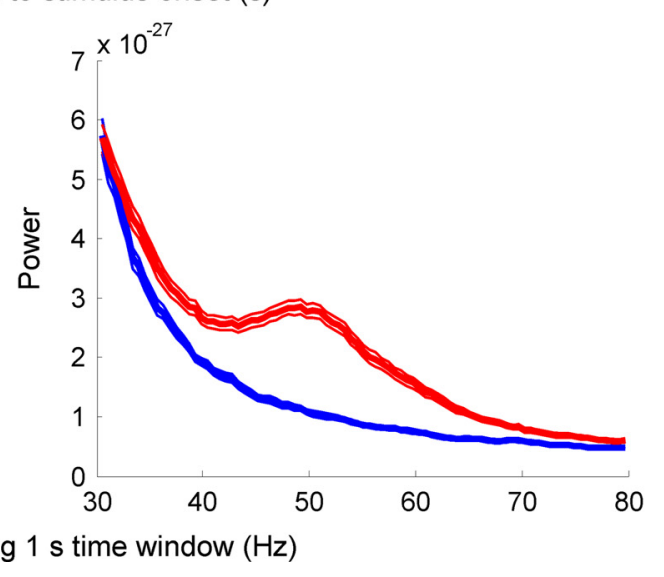

Figure 2. Gamma-band activity in two exemplar participants measured with MEG. $\boldsymbol{A}$, Time-frequency plots of oscillatory power across a trial. The intensity of the color denotes percentage power change in a frequency bin relative to the 1.5 s preceding stimulus onset (averaged across trials). $\boldsymbol{B}$, Power spectra measured from the same two participants in $\boldsymbol{A}$ in the gamma-band ( 30 - $80 \mathrm{~Hz}$ ) during a time window $0.5-1.5 \mathrm{~s}$ after stimulus onset (red) and the $1.0 \mathrm{~s}$ preceding stimulus onset (blue). Thick curves show the mean across trials; thin curves denote \pm 1 SEM.

faster gamma oscillations than static stimuli (Swettenham et al., 2009). The range of peak gamma frequencies is consistent with previous studies using similar stimulus parameters to ours (Muthukumaraswamy et al., 2009, 2010).

We further used fMRI to delineate the central part of V1 with standard retinotopic mapping techniques. As Figure 3 shows, robust statistics demonstrated a strong (Cohen, 1988) and significant positive correlation between peak gamma frequency and V1 surface area (Spearman's $\rho: \rho=0.63, p=0.011$; Fig. $3 A$; for data from individual participants, see Table 1). This relationship was evident regardless of whether V1 surface area was expressed in absolute terms or as a proportion of overall cortical surface area $(\rho=0.72, p=0.002)$. We observed the same relationship also for V2 $(\rho=0.54, p=0.03$; Fig. $3 B)$ but not for V3 $(\rho=-0.09, p=$ 0.728; Fig. $3 C$ ). No significant correlations were found between surface area and the amplitude (V1: $\rho=-0.08, p=0.782 ; \mathrm{V} 2$ : $\rho=-0.093, p=0.744)$ or width (V1: $\rho=-0.07, p=0.795$; V2: $\rho=-0.1, p=0.722$ ) of the gamma peak. Critically, the correlation between cortical thickness and gamma peak frequency was not significant (V1: $\rho=-0.03, p=0.918$; V2: $\rho=-0.118, p=$ 0.664).

Correlational analyses can be susceptible to the presence of outliers and violations of the homoscedasticity assumption. To address such possible effects, for all the correlations reported above, we calculated a robust regression by first removing outliers from the sample using the Mahalanobis distance (MD). For the correlation between V1 size and gamma frequency, one observation with $\mathrm{MD}>6$ was excluded and all results presented here are after removal of this data point. This not only ensured that potentially influential outliers did not skew the results in either direction but also guaranteed that the homoscedasticity assumption of the correlational tests was met (three different tests showed no evidence of heteroscedasticity: White's test: $p=$ 0.953, Breusch-Pagan-Koenker test: $p=0.761$, Spearman's $\rho$ : $p=0.843)$.

We restricted ourselves to only reporting the nonparametric Spearman's $\rho$ correlation, as it is more robust than the more widely used Pearson correlation. The Spearman test can also reveal nonlinear relationships that could be expected here by comparing an areal measurement, which increases within a square relationship, with a frequency, which presumably increases linearly. Similar results were obtained when using Kendall's $\tau$, another robust correlational statistic $(\tau=0.452, p=0.023)$.

We further used a bootstrapping approach to estimate the 95\% confidence interval of the correlation. We resampled the data 10,000 times with replacement and calculated the correlation coefficient for each bootstrap sample. Finally, we determined the 2.5th and 97.5th percentile of the resulting distribution of coefficients. A correlation can be considered significant if the confidence interval does not overlap zero. This was the case both when correlating gamma peak frequency with the absolute surface area of V1 (confidence interval: 0.13-0.89) and with the area relative to the surface area of the entire cortical sheet $(0.31-0.91)$. Together, these steps thus provide high confidence that there is a positive relationship between V1 surface area and gamma peak frequency. 

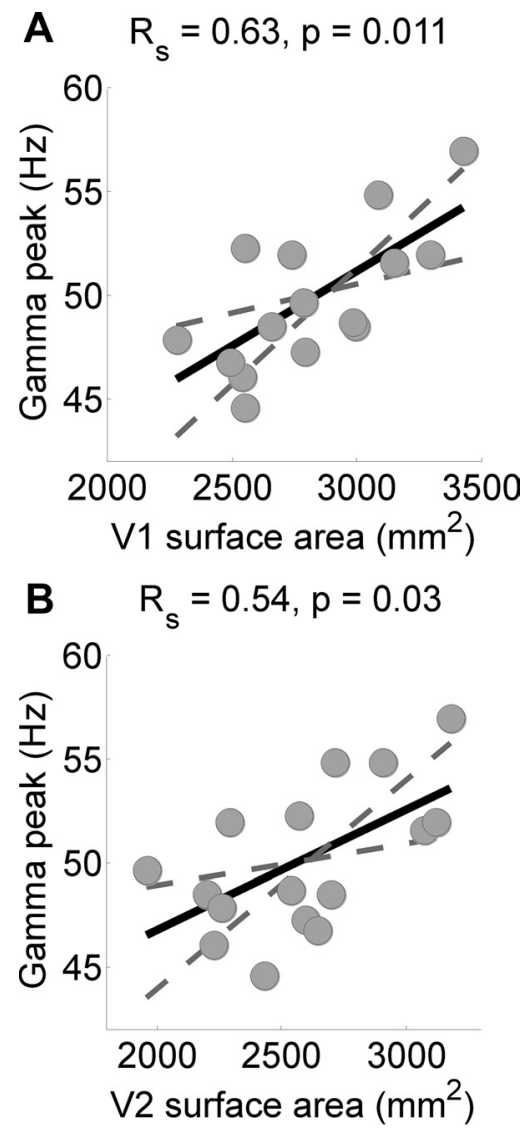

$$
\text { C } \quad R_{s}=-0.09, p=0.728
$$

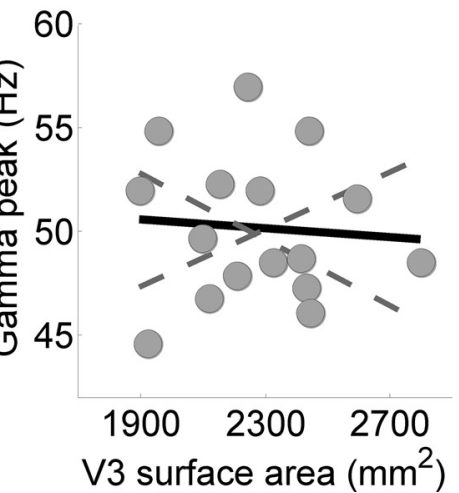

Figure 3. The retinotopically measured size of central V1 and V2 predicted individual differences in the gamma-band frequency induced by a visual stimulus. $A-C$, Gamma peak frequency in all participants plotted against the cortical surface area of V1 (A), V2 (B), and V3 (C). Each circle reflects data from one participant. The solid line is the best-fitting linear regression and dashed lines indicate bootstrapped 95\% confidence intervals. The correlation statistics (Spearman's $\rho$ ) are denoted at the top.

\section{Discussion}

Our findings provide new evidence that gamma-band frequency in human participants is correlated with the retinotopically determined surface area of central V1. The absence of a correlation with cortical thickness indicates that the effect was unrelated to gray matter volume and instead suggests that the distance across the cortical sheet was the relevant factor. This may implicate a role for lateral intra-areal connections in the generation of gamma oscillations. We propose that these individual variations in gamma frequency can also be accounted for in terms of cortical surface architecture.
Table 1. Visually induced gamma peak frequency, bandwidth, and amplitudes as well as the surface areas and cortical thickness for retinotopic areas V1-V3 in 16 participants

\begin{tabular}{|c|c|c|c|c|c|c|c|c|c|}
\hline \multirow[b]{2}{*}{ Participant } & \multicolumn{3}{|c|}{ Induced gamma } & \multicolumn{3}{|c|}{ Surface area } & \multicolumn{3}{|c|}{ Cortical thickness } \\
\hline & $\begin{array}{l}\text { Peak } \\
(\mathrm{Hz})\end{array}$ & $\begin{array}{l}\text { Bandwidth } \\
(\mathrm{Hz})\end{array}$ & $\begin{array}{l}\text { Amplitude } \\
(\%)\end{array}$ & $\begin{array}{l}\text { V1 } \\
\left(\mathrm{mm}^{2}\right)\end{array}$ & $\begin{array}{l}\text { V2 } \\
\left(\mathrm{mm}^{2}\right)\end{array}$ & $\begin{array}{l}\text { V3 } \\
\left(\mathrm{mm}^{2}\right)\end{array}$ & $\begin{array}{l}\text { V1 } \\
(\mathrm{mm})\end{array}$ & $\begin{array}{l}\text { V2 } \\
(\mathrm{mm})\end{array}$ & $\begin{array}{l}\text { V3 } \\
(\mathrm{mm})\end{array}$ \\
\hline S1 & 49.7 & 13.0 & 58.4 & 2786 & 19 & 2098 & 1.953 & 2.146 & 2.162 \\
\hline 2 & 52.0 & 13.6 & & 2736 & 2290 & & 2.048 & 2.102 & 2.145 \\
\hline 3 & 48.5 & 11.2 & & 2654 & 2699 & 2324 & 1.996 & 1.958 & 2.046 \\
\hline 4 & 48.5 & 20.5 & 46. & 2996 & 2197 & 2801 & 2.053 & 2.313 & 2.208 \\
\hline 5 & 52.3 & 16.4 & 74.6 & 2548 & 2567 & 2151 & 2.118 & 2.177 & 2.127 \\
\hline 56 & 47.3 & 10.2 & 132.6 & 2790 & 2594 & 2431 & 1.867 & 1.961 & 2.075 \\
\hline 7 & 48.7 & 11.1 & 166.7 & 2983 & 2535 & 2412 & 2.099 & 2.226 & 2.280 \\
\hline 58 & 54.9 & 11.6 & & 3083 & 2907 & 1953 & 2.020 & 2.076 & 2.163 \\
\hline 59 & 46.1 & 11.1 & 229.2 & 2539 & 2226 & 2443 & 2.285 & 2.318 & 2.291 \\
\hline S10 & 46.8 & 12.6 & 102.9 & 2488 & 2643 & 2117 & 2.216 & 2.282 & 2.220 \\
\hline S11 & 51.6 & 8.7 & 81.7 & 3149 & 3072 & 2593 & 2.122 & 2.190 & 2.356 \\
\hline S12 & 44.6 & 16.8 & 50.2 & 2550 & 2432 & 1919 & 2.079 & 2.395 & 2.490 \\
\hline S13 & 47.9 & 11.3 & 104.4 & 2277 & 2260 & 2208 & 2.079 & 2.096 & 2.347 \\
\hline S14 & 52.0 & 24.7 & 61.0 & 3295 & 3118 & 1893 & 2.351 & 2.335 & 2.409 \\
\hline S15 & 57.0 & 19.5 & 31.2 & 3425 & 3178 & 2243 & 2.062 & 2.220 & 2.344 \\
\hline S16 & 54.9 & 17.1 & 43.8 & 2112 & 2712 & 2440 & 2.180 & 2.358 & 2.501 \\
\hline
\end{tabular}

See Materials and Methods for details of how induced gamma peak and V1-V3 anatomical statistics were determined on a per-participant basis.

To explain these effects, we draw on the framework of coupled oscillators (Breakspear et al., 2010) and consider the measured gamma spectrum to be due to the summation of many synchronizing oscillators (at the scale of cortical columns) distributed across the visual cortex. Two factors influence how easily sets of coupled oscillators synchronize; their homogeneity (in frequency) and their coupling strength (connectivity) (Kuramoto, 2003). Moreover, the natural frequency of a set of spatially distributed but coupled oscillators is determined by their intrinsic coupling delay times (Jeong et al., 2002).

Consider a local pool of coupled oscillators in the visual cortex. Their ability to self-synchronize will be determined by the homogeneity in the natural frequency of their gamma oscillations. If we assume that homogeneity in environment (the similarity of the cytoarchitecture, receptor density, etc.) endows homogeneity in frequency, a local pool of oscillators will not only oscillate at similar frequencies but also will have relatively short conduction delays. That is, gamma oscillations in more homogeneous cortices should be of higher frequency for the same level of input. This is consistent with observations that neurons in more homogeneous regions of cortex have sharper orientation tuning (Nauhaus et al., 2008), that higher oscillatory frequencies are more selective to orientation (Frien et al., 2000), and that behavioral orientation discrimination is positively correlated with gamma frequency (Edden et al., 2009); i.e., higher local homogeneity gives rise to higher gamma frequency. Computationally, this is consistent with a model in which a higher gamma frequency endows a local neuronal ensemble with the ability to discriminate between shorter firing lags, thus enhancing signaling from those neurons with highest sensitivity to the stimulus (Fries et al., 2007).

Now consider adding a second (distant) pool of oscillators. As the connections to this second pool are strengthened, another form of synchrony at a lower frequency, due to the longer conduction delays, will develop. The system will have two dominant modes: local high frequency and global lower frequency. In cortices with high local homogeneity, the local neuronal populations will dominate the dynamics (as they synchronize relatively effortlessly); conversely, as the local homogeneity decreases, the dom- 
inant frequency will be determined primarily by longer-range interactions. This in turn is consistent with observations that increasing stimulus size decreases the frequency of gamma oscillation (Gieselmann and Thiele, 2008). Thus, for individuals with a large V1, where the horizontal connections traverse relatively short distances (in visual space), the homogeneity is high, and so will be the macroscopically measured oscillation frequency (as the conduction delays are short).

The relationship we propose between cortical microarchitecture and macroscopic gamma frequency may also help explain why the observed gamma frequency increases with stimulus contrast (Ray and Maunsell, 2010), as higher contrast reduces lateral signal transmission in visual cortex (Nauhaus et al., 2009), hence making the synchronized ensembles more local. Our account makes three main predictions that could be tested in future work. First, individuals with larger V1 should have better orientation tuning at the neuronal level and behaviorally better orientation discrimination ability. Second, in these individuals, gamma frequency will decrease relatively slowly (compared with people with smaller V1) as stimulus size increases. Third, at the level of cortical maps, a larger V1 should result in more homogeneous functional architecture, i.e., wider orientation columns.

Gamma-band activity and the sharpening of orientation tuning have also been linked to the activity of inhibitory neuronal networks (Alitto and Dan, 2010). Thus, the microarchitecture in V1 and inhibitory activity are likely to be closely related. In humans, the concentration of the inhibitory neurotransmitter GABA in the occipital lobe, measured using magnetic resonance spectroscopy (MRS), is correlated with the peak gammaoscillation frequency (Muthukumaraswamy et al., 2009). Previous studies showed that human early visual cortex contains a higher concentration of GABA receptors than other brain regions (Zilles et al., 2002). Histochemical studies on monkey cortex show that Brodmann's area (BA)17, which corresponds to retinotopic area $\mathrm{V} 1$, contains a considerably greater number $(\sim 50 \%$ more) of GABAergic inhibitory neurons compared with other regions, including the adjacent BA18 (Hendry et al., 1987). Assuming that the number of GABAergic cells is proportional to local GABA concentration, it is plausible that individuals with a larger V1 would have a greater concentration of GABA within a fixed volume of occipital cortex (for example, as would be measured using a fixed voxel size in MRS). But naturally, macroscopically measured GABA concentration is also likely to be influenced by factors other than cortical surface area; for example, a thicker cortex potentially contains more neurons or more GABAergic synapses. The level of GABA within cortex may also vary across individuals and even within the same individual; for example, GABA levels are likely to change across the menstrual cycle (Epperson et al., 2002). In contrast, gamma frequency has been suggested to decrease with age (Muthukumaraswamy et al., 2010; Gaetz et al., 2011). While we did not find a significant correlation between gamma frequency and age $(\rho=-0.37, p=$ 0.162 ), this may be due to the relatively narrow age range in our study (19-34 years). Our data therefore indicate that the surface area of V1 may be an important covariate to consider in any future study of both GABA levels and gamma frequency, as $\sim 36 \%$ of interindividual variance in gamma peak frequency can be explained by V1 surface area.

We also observed a significant correlation between the surface area of V2 and gamma peak frequency; while this is potentially also very interesting, it mostly likely reflects the fact that the sizes of $\mathrm{V} 1$ and $\mathrm{V} 2$ are known to be weakly correlated (Dougherty et al., 2003).
This work underlines the importance for future research to explore individual differences in the morphological organization of the cortex and to reveal the factors that drive it. It sets the way for exploring the variability of cortical functional microarchitecture and how it relates to oscillatory activity, leading to the development of mechanistic models of the underlying processes (Pinotsis and Friston, 2011).

\section{References}

Adjamian P, Holliday IE, Barnes GR, Hillebrand A, Hadjipapas A, Singh KD (2004) Induced visual illusions and gamma oscillations in human primary visual cortex. Eur J Neurosci 20:587-592.

Alitto HJ, Dan Y (2010) Function of inhibition in visual cortical processing. Curr Opin Neurobiol 20:340-346.

Bonhoeffer T, Grinvald A (1991) Iso-orientation domains in cat visual cortex are arranged in pinwheel-like patterns. Nature 353:429-431.

Breakspear M, Heitmann S, Daffertshofer A (2010) Generative models of cortical oscillations: neurobiological implications of the Kuramoto model. Front Hum Neurosci 4:190.

Cohen J (1988) Statistical power analysis for the behavioral sciences. Hillsdale, NJ: Routledge.

Dougherty RF, Koch VM, Brewer AA, Fischer B, Modersitzki J, Wandell BA (2003) Visual field representations and locations of visual areas $\mathrm{V} 1 / 2 / 3$ in human visual cortex. J Vis 3:586-598.

Duncan RO, Boynton GM (2003) Cortical magnification within human primary visual cortex correlates with acuity thresholds. Neuron 38:659-671.

Edden RA, Muthukumaraswamy SD, Freeman TC, Singh KD (2009) Orientation discrimination performance is predicted by GABA concentration and gamma oscillation frequency in human primary visual cortex. J Neurosci 29:15721-15726.

Epperson CN, Haga K, Mason GF, Sellers E, Gueorguieva R, Zhang W, Weiss E, Rothman DL, Krystal JH (2002) Cortical gamma-aminobutyric acid levels across the menstrual cycle in healthy women and those with premenstrual dysphoric disorder: a proton magnetic resonance spectroscopy study. Arch Gen Psychiatry 59:851-858.

Fischl B, Sereno MI, Dale AM (1999) Cortical surface-based analysis. II. Inflation, flattening, and a surface-based coordinate system. Neuroimage 9:195-207.

Frien A, Eckhorn R, Bauer R, Woelbern T, Gabriel A (2000) Fast oscillations display sharper orientation tuning than slower components of the same recordings in striate cortex of the awake monkey. Eur J Neurosci 12:1453-1465.

Fries P, Nikolić D, Singer W (2007) The gamma cycle. Trends Neurosci 30:309-316.

Gaetz W, Roberts TPL, Singh KD, Muthukumaraswamy SD (2011) Functional and structural correlates of the aging brain: relating visual cortex (V1) gamma band responses to age-related structural change. Hum Brain Mapp. Advance online publication. Retrieved Nov. 15, 2011. doi:10.1002/hbm.21339.

Gieselmann MA, Thiele A (2008) Comparison of spatial integration and surround suppression characteristics in spiking activity and the local field potential in macaque V1. Eur J Neurosci 28:447-459.

Hadjipapas A, Casagrande E, Nevado A, Barnes GR, Green G, Holliday IE (2009) Can we observe collective neuronal activity from macroscopic aggregate signals? Neuroimage 44:1290-1303.

Hendry SH, Schwark HD, Jones EG, Yan J (1987) Numbers and proportions of GABA-immunoreactive neurons in different areas of monkey cerebral cortex. J Neurosci 7:1503-1519.

Herrmann CS, Fründ I, Lenz D (2010) Human gamma-band activity: a review on cognitive and behavioral correlates and network models. Neurosci Biobehav Rev 34:981-992.

Hoogenboom N, Schoffelen JM, Oostenveld R, Parkes LM, Fries P (2006) Localizing human visual gamma-band activity in frequency, time and space. Neuroimage 29:764-773.

Horton JC, Hocking DR (1996) Intrinsic variability of ocular dominance column periodicity in normal macaque monkeys. J Neurosci 16:7228-7239.

Hübener M, Shoham D, Grinvald A, Bonhoeffer T (1997) Spatial relationships among three columnar systems in cat area 17. J Neurosci 17:9270-9284.

Hutton C, Bork A, Josephs O, Deichmann R, Ashburner J, Turner R (2002) 
Image distortion correction in fMRI: a quantitative evaluation. Neuroimage 16:217-240.

Jeong SO, Ko TW, Moon HT (2002) Time-delayed spatial patterns in a two-dimensional array of coupled oscillators. Phys Rev Lett 89:154104.

Kuramoto Y (2003) Chemical oscillations, waves, and turbulence. Mineola, NY: Dover.

Muthukumaraswamy SD, Edden RA, Jones DK, Swettenham JB, Singh KD (2009) Resting GABA concentration predicts peak gamma frequency and $\mathrm{fMRI}$ amplitude in response to visual stimulation in humans. Proc Natl Acad Sci U S A 106:8356-8361.

Muthukumaraswamy SD, Singh KD, Swettenham JB, Jones DK (2010) Visual gamma oscillations and evoked responses: variability, repeatability and structural MRI correlates. Neuroimage 49:3349-3357.

Nauhaus I, Benucci A, Carandini M, Ringach DL (2008) Neuronal selectivity and local map structure in visual cortex. Neuron 57:673-679.

Nauhaus I, Busse L, Carandini M, Ringach DL (2009) Stimulus contrast modulates functional connectivity in visual cortex. Nat Neurosci 12:70-76.

Pinotsis DA, Friston KJ (2011) Neural fields, spectral responses and lateral connections. Neuroimage 55:39-48.

Ray S, Maunsell JH (2010) Differences in gamma frequencies across visual cortex restrict their possible use in computation. Neuron 67:885-896.

Robinson PA (2006) Patchy propagators, brain dynamics, and the genera- tion of spatially structured gamma oscillations. Phys Rev E Stat Nonlin Soft Matter Phys 73:041904.

Schwarzkopf DS, Song C, Rees G (2011) The surface area of human V1 predicts the subjective experience of object size. Nat Neurosci 14:28-30.

Sekihara K, Nagarajan SS, Poeppel D, Marantz A (2004) Asymptotic SNR of scalar and vector minimum-variance beamformers for neuromagnetic source reconstruction. IEEE Trans Biomed Eng 51:1726-1734.

Sereno MI, Dale AM, Reppas JB, Kwong KK, Belliveau JW, Brady TJ, Rosen BR, Tootell RB (1995) Borders of multiple visual areas in humans revealed by functional magnetic resonance imaging. Science 268:889-893.

Swettenham JB, Muthukumaraswamy SD, Singh KD (2009) Spectral properties of induced and evoked gamma oscillations in human early visual cortex to moving and stationary stimuli. J Neurophysiol 102:1241-1253.

Thomson DJ (1982) Spectrum estimation and harmonic analysis. Proc IEEE 70:1055-1096.

Van Veen BD, van Drongelen W, Yuchtman M, Suzuki A (1997) Localization of brain electrical activity via linearly constrained minimum variance spatial filtering. IEEE Trans Biomed Eng 44:867-880.

Yacoub E, Harel N, Ugurbil K (2008) High-field fMRI unveils orientation columns in humans. Proc Natl Acad Sci U S A 105:10607-10612.

Zilles K, Palomero-Gallagher N, Grefkes C, Scheperjans F, Boy C, Amunts K, Schleicher A (2002) Architectonics of the human cerebral cortex and transmitter receptor fingerprints: reconciling functional neuroanatomy and neurochemistry. Eur Neuropsychopharmacol 12:587-599. 